Yoshihei Hasegawa

Nagoya Math. J.

Vol. 40 (1970), 67-84

\title{
G-FLOWS ON A LIE GROUP FOR EULER EQUATIONS
}

\author{
YOSHIHEI HASEGAWA
}

\section{§ 0. Introduction}

The purpose of this paper is to determine left-invariant vector fields on a Lie group $G$ with a left-invariant Riemannian metric which induces $C$ flows on $G$.

In his paper [1], V.I. Arnol'd has obtained a differential equation (Euler equation) analogous to that of motion of a rigid body about a fixed point under no forces. Let $\left\{g_{t} ; t \in R\right\}$ be a geodesic on a Lie group $G$ with a left-invariant Riemannian metric and let $\$$ be its Lie algebra. Then

$$
X_{t}=\left(L_{g_{t}}^{-1}\right)_{*} g_{t} \in \mathbb{S S}
$$

satisfies the following differential equation (Euler equation),

$$
\dot{X}_{t}=B\left(X_{t}, X_{t}\right),
$$

where $B$ is a bilinear map of $\mathbb{E} \times \mathbb{S}$ into $\mathbb{S}$ depending on the Riemannian metric.

In particular, Arnol'd has paid attention to the geodesic expressed as a one-parameter subgroup of $G$, which is analogous to a closed (periodic) geodesic, to study the stability of the stationary points $X(B(X, X)=0)$ of the equation (2) in \&s rather than the stability of the corresponding geodesics of one-parameter subgroups.

In connection with ergodic theory, we are interesed in the exact form of the Euler equation with a particular choice of the Lie group. Namely, we consider the Euler equation on the projective special linear group PSL (2. $R$ ) of 2-nd order over $R$ with the natural left-invariant Riemannian metric, and determine its instable stationary points. Then we shall see that each instable stationary point induces a $C$-flow on $P S L(2 . R)$ in our sense (Cor. 3.1.).

Being inspired by the remarkable property above, we shall determine left-invariant vector fields, each of which induces a $C$-flow on a Lie group.

Received June 30, 1969. 
Our main results read as follows. We employ the usual notations such as $A d$ and $a d$ to denote the adjoint representation of a Lie group and the adjoint representation of its Lie algebra.

Theorem 2. Let $G$ be an n-dimensional oriented, connected real Lie group with the unit element $e$, and let $\mathbb{\&}$ be its Lie algebra. Assume that $\mathbb{1 S}$ contains an element $X$ satisfying the following conditions:

1) $a d(X)$ is diagonal on the complexification $\mathbb{S}^{c}$ of $\&$.

2) The multiplicity of the eigenvalue 0 is exactly equal to 1.

3) The rest of the eigenvalues of ad $(X)$ is divided into two parts, call them $\lambda_{1}, \cdots, \lambda_{k} ; \mu_{1} \cdots \mu_{l}$, in such a way that

$$
\begin{aligned}
& \text { Re } \lambda_{i}>0, i=1, \cdots, k, \\
& \text { Re } \mu_{i}<0, i=1, \cdots, l,
\end{aligned}
$$

where $k \geq 1, l \geq 1$ and $k+l+1=n$.

4) $\operatorname{Tr} \operatorname{ad}(X)=0$.

Then the one-parameter group of the diffeomorphisms $\operatorname{Expt} X$ of $G$ is a $C$-flow with respect to any left-invariant Riemannian metric $d s^{2}$ on $G$.

If we specify the Lie group to be semi-simple, we shall have the following

Theorem 4. The semi-simple real Lie algebra \&s is isomorphic to $\mathfrak{I l}(2 . R)$, if (8) contains an element satisfying the conditions 1),2), 3), 4) of Theorem 2.

Observing the above-mentioned situation, we are led to pay specific attention to $P S L(2 . R)$ and to transform $C$-flows on $P S L(2 . R)$ formed as above to the unitary tangent bundle $T_{1} L$ of the Lobachevsky-plane $L$. There we shall see an interesting result:almost all $C$-flows thus transformed can not be geodesic flows on the upper half-plane respect to any Riemannian metric (Theorem 5).

Finally, the author hopes that our approach to the stability of a stationary point of the equation (2) will be useful in the investigation of the stability of a stationary current for Euler equation appearing in the hydrodynamics on a Riemannian manifold (see Arnol'd [1]).

The author wishes to express his deepest gratitude to the members of the seminar on probability at Nagoya University. 


\section{$\S 1$. Notations and Definitions}

We shall list some notations and give definitions used in the later sections.

Let $G$ be a real connected Lie group and let \&s be its Lie algebra over real numbers $R$ with the bracket [, ]. Assume that $G$ has a left-invariant Riemannian metric $d s^{2}$ i.e. the pull-back $L_{g}^{*} d s^{2}$ of $d s^{2}$ by any left-translation $L_{g}(g \in G)$ is equal to $d s^{2}$. Then an inner product $\langle$,$\rangle is naturally induced$ in \&s from $d s^{2}$. By means of this inner product, we define a bilinear map $B$ :

$$
B: \circlearrowleft 5 \times \mathbb{S} \ni(X, Y) \longrightarrow B(X, Y) \in \mathscr{S}
$$

of the product space $\mathfrak{B S} \times \mathbb{S}$ into $\mathbb{S}$ as follows:

$$
\langle B(X, Y), Z\rangle=\langle[Y, Z], X\rangle \text { for } \quad X, Y, Z \in \mathbb{S} \text {. }
$$

Now let us consider the following differentisl equation for $C^{1}$-curve $\{X(t) \in \mathbb{C} ; t \in R\}$ :

$$
\left.\frac{d}{d t} X(t)\right|_{t=s}=B(X(s), X(s))
$$

This equation is called the Euler equation on $G$ (or on (S) associated with the left-invariant Riemannian metric $d s^{2}$.

Next let us consider the following differential equation in the $n$ dimensional vector space $R^{n}$ :

$$
\left.\frac{d}{d t} X(t)\right|_{t=s}=f(X(s)), X(s) \in R^{n} .
$$

Then we can think of the equation (3) as the expression of the infinitesimal transformation on $R^{n}$. A point $X_{0} \in R^{n}$ is said to be stationary if $f\left(X_{0}\right)=0$. A stationary point $X_{0}$ is said to be stable if $X_{0}$ satisfies the following conditions: Let $X(t)$ be the solution of

$$
\begin{aligned}
& \left.\frac{d}{d t} X(t)\right|_{t=s}=f(X(s)) \\
& X(0)=X .
\end{aligned}
$$

Then, for any $\varepsilon>0$, there exists $\delta>0$ such that

$$
\left\|X(t)-X_{0}\right\|<\varepsilon \text { for any } t \geq 0 \text { and any } X \in R^{n} \text { with }\left\|X-X_{0}\right\|<\delta,
$$


where

$$
\left\|\left(x_{1}, \cdots, x_{n}\right)\right\|=\max \left\{\left|x_{i}\right| ; i=1, \cdots, n\right\} .
$$

If a stationary point is not stable, it is said to be instable.

Remark. The uniqueness of the solution of the equation (2) is guaranteed in our case. It should be noted that we are always given the solutoin $X(t)$ for which (2) holds for all $s$.

\section{§2. Euler equation on the projective special linear group PSL} (2.R) of second order

In this section we construct Euler equation on the Lie group PSL (2.R) with some natural left-invariant Riemannian metric, and determine the stability of its stationary points.

Let us list some notations.

The Lobachevsky-plane is denoted by $\left(L, d s_{1}^{2}\right)$ i.e.

$$
L=\{(x, y) \mid x \in R, y>0\}, d s_{1}^{2}=\frac{d x^{2}+d y^{2}}{y^{2}}
$$

A tangent vector $v$ at a point $(x, y) \in L$ is expressex as follows:

$$
v=-h y \sin \theta\left(\frac{\partial}{\partial x}\right)_{(x, y)}+h y \cos \theta\left(\frac{\partial}{\partial y}\right)_{(x, y)},
$$

where $h \geq 0$ and $-\pi \leq \theta<\pi$.

The vector $v_{0}$ always means

$$
v_{0}=\left(\frac{\partial}{\partial y}\right)_{(0.1)}
$$

We denote by $g$ an isometry on $L$ induced from the element $g \in P S L(2 . R)$ under the natural identification of $P S L(2 . R)$ with the isometries on $L$. Under this identification, we define a diffeomorphism $\Phi$ of the unitary tangent bundle $T_{1} L$ (see [2] for definition) of $L$ onto PSL (2.R) as follows:

$$
\Phi\left(g_{*} v_{0}\right)=g \in P S L(2 . R),
$$

where $g_{*}$ denotes the differential of the isometry $g$ on $L$. By means of the following unique decomposition of elements of PSL (2.R), we parametrize $P S L(2 . R)$ 


$$
(x, y, \theta)= \pm\left(\begin{array}{ll}
1 & x \\
0 & 1
\end{array}\right)\left(\begin{array}{cc}
\sqrt{y} & 0 \\
0 & \frac{1}{\sqrt{y}}
\end{array}\right)\left(\begin{array}{cc}
\cos \theta & \sin \theta \\
-\sin \theta & \cos \theta
\end{array}\right)
$$

where

$$
x \in R, y>0,-\frac{\pi}{2} \leq \theta<\frac{\pi}{2} .
$$

With these notions the following propositions can easily be proved.

Proposition 2.1. Making use of the parameters $(x, y, \theta)$ in $T_{1} L$ and the parameters $(x, y, \theta)$ in PSL (2.R), the diffeomorphism $\Phi$ of $T_{\mathfrak{l}} L$ onto PSL $(2 . R)$ is expressed as follows:

$$
\Phi: T_{1} L \ni(x, y, \theta) \longrightarrow\left(x, y, \frac{\theta}{2}\right) \in P S L(2 . R) .
$$

Proposition 2.2. The Riemannian metric $d s_{2}^{2}$ on $T_{1} L$ induced from $d s_{1}^{2}$ on $T_{1} L$ in the usual manner is expressed in the form

$$
d s_{2}^{2}=\frac{2}{y^{2}} d x^{2}+\frac{1}{y^{2}} d y^{2}+\frac{2}{y} d x d \theta+d \theta^{2} .
$$

Proposition 2.3. The Riemannian metric $d s_{2}^{2}$ on $T_{1} L$ induces by $\Phi^{-1}$ the following Riemannian metric $d s^{2}$ on PSL (2.R)

$$
d s^{2}=\left(\Phi^{-1}\right)^{*} d s_{2}^{2}=\frac{2}{y^{2}} d x^{2}+\frac{1}{y^{2}} d y^{2}+\frac{4}{y} d x d \theta+4 d \theta^{2}
$$

Proposition 2.4. The above Riemannian metric $d s^{2}$ on PSL $(2 . R)$ is leftinvariant:

$$
L_{g}^{*} d s^{2}=d s^{2} \quad \text { for } \quad g \in P S L(2 . R) .
$$

(Proof) Let $g, h$ be elements of PSL (2.R) with the unit element" $e$. Then by the definition of $\Phi$,

$$
\begin{aligned}
& g_{*} h_{*} v_{0}=(g \cdot h)_{*} v_{0}=\Phi^{-1}(g \cdot h)=\Phi^{-1} L_{g, h} e \\
& =\Phi^{-1} L_{g} \Phi \Phi^{-1} L_{h} e=\Phi^{-1} L_{g} \Phi h_{*} v_{0},
\end{aligned}
$$

that is,

$$
\Phi^{-1} L_{g}=g_{*} \Phi^{-1}
$$


This implies

$$
\Phi_{*}^{-1}\left(L_{g}\right)_{*}=\left(g_{*}\right)_{*} \Phi_{*}^{-1} \quad \text { for } \quad g \in P S L(2 . R) \text {. }
$$

Therefore, denoting by $\|\cdot\|$ the norn given by $d s^{2}$ and by $\|\cdot\|^{\prime}$ the norm given by $d s_{2}^{2}$, the following equalities hold for any tangent vector $v$ of $P S L(2 . R)$ and any element $g \in P S L(2 . R)$ :

$$
\left\|\left(L_{g}\right)_{*} v\right\|=\left\|\Phi_{*}^{-1}\left(L_{g}\right)_{*} v\right\|^{\prime}=\left\|\left(g_{*}\right)_{*} \Phi_{*}^{-1} v\right\|^{\prime}=\left\|\Phi_{*}^{-1} v\right\|^{\prime}=\|v\|,
$$

which complete the proof.

The Lie algebra of $P S L(2 . R)$, as is well known, coincides with $\mathfrak{g l}(2 . R)$. We now introduce the following left-invariant vector fields $X_{1}, X_{2}, X_{3}$ given by

$$
\left(X_{1}\right)_{e}=\left(\frac{\partial}{\partial x}\right)_{e}, \quad\left(X_{2}\right)_{e}=2\left(\frac{\partial}{\partial y}\right)_{e}, \quad\left(X_{3}\right)_{e}=\left(\frac{\partial}{\partial \theta}\right)_{e}
$$

Then we can easily prove the following

Proposition 2.5. The commutation-relations for $X_{1}, X_{2}, X_{3}$ are expressed in thr form

$$
\begin{aligned}
& {\left[X_{1}, X_{2}\right]=-2 X_{1},} \\
& {\left[X_{2}, X_{3}\right]=-2 X_{3}+4 X_{1},} \\
& {\left[X_{3}, X_{1}\right]=X_{2} .}
\end{aligned}
$$

By the definition of the inner product and with the choice of these $X_{i}^{\prime}$ s we are given the following formula

$$
\begin{aligned}
& \left\langle\xi_{1} X_{1}+\xi_{2} X_{2}+\xi_{3} X_{3}, \eta_{1} X_{1}+\eta_{2} X_{2}+\eta_{3} X_{3}\right\rangle \\
= & 2 \xi_{1} \eta_{1}+4 \xi_{2} \eta_{2}+4 \xi_{3} \eta_{3}+2 \xi_{1} \eta_{3}+2 \xi_{3} \eta_{1},
\end{aligned}
$$

where $\xi_{1}, \xi_{2}, \xi_{3}, \eta_{1}, \eta_{2}, \eta_{3}$ are all real numbers.

By virtue of the propositions above, we obtain

Theorem 1. The Euler equation (2) on $\mathfrak{I l}(2 . R)$ associated with the leftinvariant Riemannian metric $d s^{2}$ is expressed as follows:

$$
\left\{\begin{array}{l}
\dot{\xi}_{1}(t)=4 \xi_{2}(t)\left(\xi_{1}(t)+2 \xi_{3}(t)\right) \\
\dot{\xi}_{2}(t)=-\xi_{1}(t)\left(\xi_{1}(t)+2 \xi_{3}(t)\right) \\
\dot{\xi}_{3}(t)=-2 \xi_{2}(t)\left(\xi_{1}(t)+2 \xi_{3}(t)\right),
\end{array}\right.
$$

where 


$$
X(t)=\xi_{1}(t) X_{1}+\xi_{2}(t) X_{2}+\xi_{3}(t) X_{3}
$$

The stationary points $X$ of this Euler equation are expressed in the form

$$
X=\xi_{3} X_{3} \quad\left(\xi_{3} \in R\right),
$$

or

$$
X=-2 \xi_{3} X_{1}+\xi_{2} X_{2}+\xi_{3} X_{3} \quad\left(\xi_{2}, \xi_{3} \in R\right) .
$$

Further, the stationary points $X=\xi_{3} X_{3}$ are stable, while the stationary points $X=-2 \xi_{3} X_{1}+\xi_{2} X_{2}+\xi_{3} X_{3}\left(\xi_{2}^{2}+\xi_{3}^{2} \neq 0\right)$ are instable.

(Proof) Let $X, X^{\prime}$ be elements of $\mathfrak{g} \mathfrak{j}(2 . R)$ :

$$
\begin{aligned}
& X=\xi_{1} X_{1}+\xi_{2} X_{2}+\xi_{3} X_{3} \\
& X^{\prime}=\xi_{1}^{\prime} X_{1}+\xi_{2}^{\prime} X_{2}+\xi_{3}^{\prime} X_{3} .
\end{aligned}
$$

Then by Proposition 2.5,

$$
\begin{aligned}
{\left[X, X^{\prime}\right]=} & 2\left(\xi_{2} \xi_{1}^{\prime}-\xi_{1} \xi_{2}^{\prime}+2 \xi_{2} \xi_{3}^{\prime}-2 \xi_{3} \xi_{2}^{\prime}\right) X_{1} \\
& +\left(\xi_{3} \xi_{1}^{\prime}-\xi_{1} \xi_{3}^{\prime}\right) X_{2}+2\left(\xi_{3} \xi_{2}^{\prime}-\xi_{2} \xi_{3}^{\prime}\right) X_{3} .
\end{aligned}
$$

Hence we have by Proposition 2.5,

$$
\left\langle\left[X, X^{\prime}\right], X\right\rangle=4\left(\xi_{1}+2 \xi_{3}\right) \xi_{2} \xi_{1}^{\prime}-4\left(\xi_{1}+2 \xi_{3}\right) \xi_{1} \xi_{2}^{\prime} .
$$

Set

$$
B(X, X)=\eta_{1} X_{1}+\eta_{2} X_{2}+\eta_{3} X_{3}
$$

then we have

$$
\left\langle B(X, X), X^{\prime}\right\rangle=2\left(\eta_{1}+\eta_{3}\right) \xi_{1}^{\prime}+4 \eta \xi_{2}^{\prime}+2\left(\eta_{1}+2 \eta_{3}\right) \xi_{3}^{\prime} .
$$

By the definition of the map $B$ we have

$$
\left\langle B(X, X), X^{\prime}\right\rangle=\left\langle\left[X, X^{\prime}\right], X\right\rangle .
$$

Hence

$$
\left\{\begin{array}{l}
\eta_{1}=4 \xi_{2}\left(\xi_{1}+2 \xi_{3}\right) \\
\eta_{2}=-\xi_{1}\left(\xi_{1}+2 \xi_{3}\right) \\
\eta_{3}=-2 \xi_{2}\left(\xi_{1}+2 \xi_{3}\right) .
\end{array}\right.
$$

Consequently, we obtain the following expression: 


$$
\left\{\begin{array}{l}
\dot{\xi}_{1}(t)=4 \xi_{2}(t)\left(\xi_{1}(t)+2 \xi_{3}(t)\right) \\
\dot{\xi}_{2}(t)=-\xi_{1}(t)\left(\xi_{1}(t)+2 \xi_{3}(t)\right) \\
\dot{\xi}_{3}(t)=-2 \xi_{2}(t)\left(\xi_{1}(t)+2 \xi_{3}(t)\right)
\end{array}\right.
$$

where

$$
X(t)=\xi_{1}(t) X_{1}+\xi_{2}(t) X_{2}+\xi_{3}(t) X_{3} .
$$

Now let us introduce a new variable $\zeta$ by

$$
\zeta=\xi_{1}+2 \xi_{3} \text {. }
$$

Then the Euler equation turns out to be

$$
(*)\left\{\begin{array}{l}
\dot{\xi}_{1}(t)=4 \xi_{2}(t) \zeta(t) \\
\dot{\xi}_{2}(t)=-\xi_{1}(t) \zeta(t) \\
\dot{\zeta}(t)=0
\end{array}\right.
$$

This implies

$$
\left\{\begin{array}{l}
\xi_{1}(t)=\xi_{1}(0) \cos 2 \zeta(0) t+2 \xi_{2}(0) \sin 2 \zeta(0) t \\
\xi_{2}(t)=-\frac{\xi_{1}(0)}{2} \sin 2 \zeta(0) t+\xi_{2}(0) \cos 2 \zeta(0) t \\
\zeta(t)=\zeta(0) .
\end{array}\right.
$$

Hence

$$
\left\{\left(\xi_{1}=0, \xi_{2}=0, \zeta=w\right) ; w \in R\right\} \cup\left\{\left(\xi_{1}=u, \xi_{2}=v, \zeta=0\right) ; u^{2}+v^{2} \neq 0, u, v \in R\right\}
$$

is the set of all the stationary points of the equation $(*)$, and further it is easily seen that the stationary point $\left(\xi_{1}=0, \xi_{2}=0, \zeta=w\right)$ is stable for the equation $(*)$, while the stationary point $\left(\xi_{1}=u, \xi_{2}=v, \zeta=0\right)\left(u^{2}+v^{2} \neq 0\right)$ is inatsble for the equation $(*)$.

The following linear map of 3-dimensional vector space $R^{3}$ into itself is regular,

$$
R^{3} \ni\left(\xi_{1}, \xi_{2}, \xi_{3}\right) \longrightarrow\left(\xi_{1}, \xi_{2}, \xi_{1}+2 \xi_{3}\right) \in R^{3} .
$$

Summing up the above results, we obtain the conclusions.

(Q.E.D.) 


\section{$\S 3$. The left-invariant vector fields which induce $C$-flows on a connected real Lie group}

Let $G$ be a connected real Lie group with a left-invariant Riemannian metric. We give a sufficient condition for a left-invariant vector field on $G$ to induce a $C$-flow on it in our sense (Theorem 2). We prove that if, in particular, the Lie group \&\& is semi-simple, then its Lie algebra \&\& is isomorphic to $\mathfrak{i l}(2 . R)$ (Theorem 4 ).

We begin with the definitions of $C$-flows in our sense.

Definition. Let $\left\{\varphi_{t} ; t \in R\right\}$ be a one-parameter group of $C^{2}$-diffeomorphisms of $n$-dimensional oriented, connected $C^{\infty}$-manifold $M$ with a Riemannian metric $d s^{2}$.

If the following conditions are satisfied, $\varphi_{t}$ is called a $C$-flow:

1) The infinitesimal transformation $X$ of $\varphi_{t}$ vanishes nowhere, and the divergence of $X$ vanishes everywhere.

2) The tangent vector space $T M_{x}$ at $x \in M$ splits into a direct sum:

$$
T M_{x}=A_{x} \oplus B_{x} \oplus C_{x},
$$

where $A_{x}$ and $B_{x}$ are vector subspaces with $\operatorname{dim} A_{x}=k \geq 1, \operatorname{dim} B_{x}=l \geq 1$, and where $C_{x}$ is the 1-dimentional subspace spanned by $X_{x}$.

3) For any $v \in A_{x}$

$$
\begin{aligned}
& \left\|\left(\varphi_{t}\right)_{*} v\right\| \geqq a e^{\lambda t}\|v\|, t \geq 0, \\
& \left\|\left(\varphi_{t}\right)_{*} v\right\| \leqq b e^{\lambda t}\|v\|, t \leq 0 ;
\end{aligned}
$$

and for any $v \in B_{x}$

$$
\begin{aligned}
& \left\|\left(\varphi_{t}\right)_{*} v\right\| \leqq b e^{-\lambda t}\|v\|, t \geq 0, \\
& \left\|\left(\varphi_{t}\right)_{*} v\right\| \geqq a e^{-\lambda t}\|v\|, t \leq 0,
\end{aligned}
$$

where $\|\cdot\|$ denotes the norm given by $d s^{2}$ and where $a, b, \lambda$ are positive constants.

We denote by $A d$ and ad the adjoint representation of a Lie group and the adjoint representation of its Lie algebra respectively.

Theorem 2. Let $G$ be a n-dimensional oriented connected real Lie group with the unti element $e$, and let $\&$ be its Lie algebra. Assume that $\&$ sontains an element $X$ satisfying the following conditions:

The term "C-flow" is usually used only in the case where the manifold is compact. 
1) $\operatorname{ad}(X)$ is diagonal on the complexification $\mathbb{S S}^{c}$ of 85.

2) The multiplicity of the eigenvalue 0 is exactly equal to 1.

3) The rest of the eigenvalues of $a d(X)$ is divided into two parts, call them $\lambda_{1}, \cdots, \lambda_{k} ; \mu_{1}, \cdots, \mu_{l}$, in such a way that

$$
\begin{aligned}
& \operatorname{Re} \lambda_{i}>0, i=1, \cdots, k, \\
& \operatorname{Re} \mu_{i}<0, i=1, \cdots, l,
\end{aligned}
$$

where $k \geq 1, l \geq 1$ and $k+l+1=n$.

4) $\operatorname{Tr} a d(X)=0$.

Then the one-parameter group of the diffeomorphisms Expt $X$ of $G$ is a C-flow with respect to any left-invariant Riemannian metric $d s^{2}$ on $G$.

(Proof) $1^{0}$. By. the assumption 3) we can express $\left\{\lambda_{i}\right\}$ in the form

$$
\begin{aligned}
& \lambda_{i}=\alpha_{i}+\sqrt{-1} \beta_{i}, \quad i=1, \cdots, p, p \geqq 0 \\
& \lambda_{i+p}=\alpha_{i}-\sqrt{-1} \beta_{i}, \quad \alpha_{i}>0, \beta_{i}>0 \\
& \lambda_{i}>0, \quad i=2 p+1, \cdots, k .
\end{aligned}
$$

Since $a d(X)$ is diagonal on \&S $^{c}$, there exist $Z_{i}(\neq 0)$ in \&s such that

$$
\operatorname{ad}(X) Z_{i}=-\lambda_{i} Z_{i}, i=2 p+1, \cdots, k,
$$

that is,

$$
\exp (-\operatorname{tad}(X)) Z_{i}=e^{\lambda_{i} t} Z_{i} i=2 p+1, \cdots, k .
$$

Similarly there exist $X_{i}(\neq 0), Y_{i}(\neq 0)$ in \&s such that

$$
a d(X)\left(X_{i}+\sqrt{-1} Y_{i}\right)=-\left(\alpha_{i}+\sqrt{-1} \beta_{i}\right)\left(X_{i}+\sqrt{-1} Y_{i}\right) i=1, \cdots, p .
$$

Therefore we have

$$
\exp (-t \operatorname{ad}(X))\left(X_{i}+\sqrt{-1} Y_{i}\right)=e^{\left(\alpha_{i}+\sqrt{-1} \beta_{i}\right) t}\left(X_{i}+\sqrt{-1} Y_{i}\right)
$$

which implies

$$
\begin{aligned}
& \exp (-t a d(X)) X_{i}=e^{\alpha_{i} t}\left(\cos \beta_{i} t X_{i}-\sin \beta_{i} t Y_{i}\right) \\
& \exp (-t a d(X)) Y_{\imath}=e^{\alpha_{i} t}\left(\cos \beta_{i} t Y_{i}+\sin \beta_{i} t X_{i}\right) .
\end{aligned}
$$

On the other hand, since $\beta_{i} \neq 0, X_{i}$ and $Y_{i}$ are $R$-lineraly independent. Hence the collection $\left\{X_{i}, Y_{i}, Z_{i}\right\}$ spans a $k$-dimensional subspace $A$ of $\mathbb{S}$.

Now for any combination of real numbers 


$$
\left\{x_{i}, y_{i} ; i=1, \cdots, p\right\} \cup\left\{z_{i} ; i=2 p+1, \cdots, k\right\},
$$

we define $Y \in \&$ by

$$
Y=\sum_{i=1}^{p} x_{i} X_{i}+\sum_{i=1}^{p} y_{i} Y_{i}+\sum_{i=2 p+1}^{k} z_{i} Z_{i} .
$$

Then we have

$$
\begin{aligned}
\exp (-t \operatorname{ad}(X)) Y= & \sum_{i=1}^{p} e^{\alpha_{i} t} \rho_{i}\left(\cos \left(\beta_{i} t+\theta_{i}\right) X_{i}+\sin \left(\beta_{i} t+\theta_{i}\right) Y_{i}\right) \\
& +\sum_{i=2 p+1}^{k} z_{i} e^{\lambda_{i} t} Z_{i},
\end{aligned}
$$

where $\rho_{i}, \theta_{i}(i=1, \cdots, p)$ are determined by the following formulas,

$$
x_{i}=\rho_{i} \cos \theta_{i}, \quad y_{i}=\rho_{i} \cos \theta_{i}, \quad \rho_{i} \geq 0,0 \leq \theta_{i}<2 \pi .
$$

For a moment, we introduce an inner product in $A$ so that $\left\{X_{i}, Y_{i}, Z_{i}\right\}$ forms a complete orthonormal system in $A$, and denote by $\|\cdot\|^{\prime}$ the norm given by this inner product. Further we define $l(t)$ by

$$
l(t)^{2}=\|\exp (-t a d(X)) Y\|^{\prime 2} .
$$

Then it holds that

$$
l(t)^{2}=\sum_{i=1}^{p} e^{2 \alpha_{i} t} \rho_{i}^{2}+\sum_{i=2 p+1}^{k} e^{2 \alpha_{i} t} z_{i}^{2} .
$$

Hence we have

$$
\begin{aligned}
& l(t)^{2} \geq e^{2 \nu t} l(0)^{2}, \quad t \geq 0, \\
& l(t)^{2} \leqq e^{2 \nu} l(0)^{2}, \quad t \leq 0,
\end{aligned}
$$

where

$$
\nu=\min \left\{\operatorname{Re} \lambda_{i} ; i=1, \cdots, k\right\} .
$$

It is easily seen that there exist positive numbers $a_{1}, b_{1}$ for which the following inequalities hold:

$$
\begin{aligned}
& \| \exp \left(-t \operatorname{ad}(X) Y\left\|\geq a_{1} e^{\nu t}\right\| Y \|, t \geq 0,\right. \\
& \| \exp \left(-t a d(X) Y\left\|\leq b_{1} e^{\nu t}\right\| Y \|, t \leq 0, \text { for any } Y \in A,\right.
\end{aligned}
$$

where $\|\cdot\|$ denotes the original norm.

Denoting by $B$ the $l$-dimensional subspace of $\&$ corresponding to the eigen- 
values $\mu_{1}, \cdots, \mu_{l}$, we can, in a similar manner, find numbers $a_{2}, b_{2}$ such that

$$
\begin{aligned}
& \| \exp (-t \text { ad }(X)) Y\left\|\leq b_{2} e^{-\mu t}\right\| Y \|, t \geq 0, \\
& \| \exp (-t \text { ad }(X)) Y\left\|\geqq a_{2} e^{-\mu} t\right\| Y \|, t \leq 0, \text { for any } Y \in B,
\end{aligned}
$$

where

$$
-\mu=\max \left\{\operatorname{Re} \mu_{i} ; i=1, \cdots, l\right\} .
$$

Consequently there exist positive numbers $a, b$ such that the following two pairs of inequalities hold simultaneously:

$$
\begin{aligned}
& \|\exp (-t \operatorname{ad}(X)) Y\| \geqq a e^{\lambda t}\|Y\|, t \geqq 0, \\
& \|\exp (-t \operatorname{ad}(X)) Y\| \leqq b e^{\lambda t}\|Y\|, \quad t \leq 0, \text { for any } Y \in A,
\end{aligned}
$$

and

$$
\begin{aligned}
& \|\exp (-t \operatorname{ad}(X)) Y\| \leqq b e^{-\lambda t}\|Y\|, t \geqq 0, \\
& \|\exp (-t a d(X)) Y\| \geqq a e^{-\lambda t}\|Y\|, t \leqq 0, \text { for any } Y \in B,
\end{aligned}
$$

where

$$
\lambda=\min \{\nu, \mu\} \text {. }
$$

$2^{0}$. We are given by $d s^{2}$ the inner product $\langle,\rangle_{h}$ and norm $\|\cdot\|_{h}$ (or simply $\langle\rangle,,\|\cdot\|)$ in the tangent vector space $T G_{h}$ at $h \in G$. Let $Y$ be an element of $A$, and let $h$ be an element of $G$. Then, by the left-invariance of $d s^{2}$, we obtain the following formulas:

$$
\begin{aligned}
& \left\|(\operatorname{Exp} t X)_{*} Y_{h}\right\|=\left\|\left(L_{h}^{-1}\right)_{*}\left(R_{\exp t X}\right)_{*} Y_{h}\right\|=\left\|\left(R_{\exp t X}\right)_{*} Y_{e}\right\| \\
= & \left\|(L \exp (-t X))_{*}\left(\operatorname{Rexp}_{t X}\right)_{*} Y_{e}\right\|=\|A d \exp (-t X) Y\| \\
= & \|\exp (-t \operatorname{ad}(X)) Y\| .
\end{aligned}
$$

Hence we have

$$
\begin{aligned}
& \left\|(\operatorname{Exp} t X)_{*} Y_{h}\right\| \geqq a e^{\lambda t}\|Y\|=a e^{\lambda t}\left\|Y_{h}\right\|, t \geqq 0 \\
& \left\|(\operatorname{Exp} t X)_{*} Y_{h}\right\| \leqq b e^{\lambda t}\|Y\|=b e^{\lambda t}\left\|Y_{h}\right\|, t \leq 0 \text { for any } Y \in A .
\end{aligned}
$$

For any element $h \in G$, we can also prove the following formulas:

$$
\begin{aligned}
& \left\|(\operatorname{Exp} t X)_{*} Y_{h}\right\| \leqq b e^{-\lambda t}\left\|Y_{h}\right\|, t \geqq 0, \\
& \left\|(\operatorname{Exp} t X)_{*} Y_{h}\right\| \geqq a e^{-\lambda t}\left\|Y_{h}\right\|, t \leqq 0 \text { for any } Y \in B .
\end{aligned}
$$


$3^{0}$ For a vector field $Z$ on $G$ we define a one-form $\omega_{Z}$ on $G$ as follows:

$$
\omega_{Z}(\cdot)=\langle\cdot, Z\rangle .
$$

Let $\left\{W_{1}, \cdots, W_{n}\right\}$ be the orthonormal basis in $\mathbb{S}$. Then

$$
\Omega=\omega_{W_{1}} \wedge \cdots \wedge \omega_{W_{n}}
$$

is a volume element on $G$.

Now let $g, h$ be elements of $G$, and let $v$ be an element of $T G_{h}$. Then we have

$$
\begin{aligned}
&\left(L_{g}^{*} \omega_{W_{i}}\right)_{h}(v)=\left(\omega_{W_{i}}\right)_{g \cdot h}\left(L_{g^{*}} v\right)=\left\langle\left(W_{i}\right)_{g \cdot h}, L_{g^{*} v}\right\rangle \\
&=\left\langle L_{g^{*}}^{-1}\left(W_{i}\right)_{g \cdot h}, v\right\rangle=\left\langle\left\langle W_{i}\right)_{h}, v\right\rangle=\left(\omega_{W_{i}}\right)_{h}(v),
\end{aligned}
$$

that is,

$$
L_{g}^{*} \omega_{W_{i}}=\omega_{W_{i}} \cdot
$$

Hence, for any element $Y \in \mathbb{S}$ it holds that

$$
\begin{aligned}
& \left((\operatorname{Exp} t X)^{*} \omega_{W_{t}}\right)(Y)=\left(L_{\exp (-t x)}^{*} R_{\exp t X}^{*} \omega_{W_{i}}\right)(Y) \\
& =\left\langle W_{i}, A d_{\exp (-t X)} Y\right\rangle=\left\langle W_{i}, \exp (-t \operatorname{ad}(X)) Y\right\rangle .
\end{aligned}
$$

Hence we have

$$
\begin{aligned}
\left(L_{X} \omega_{W_{i}}\right)(Y) & =\lim _{t \rightarrow 0} \frac{(\operatorname{Exp} t X)^{*} \omega_{W_{t}}-\omega_{W_{i}}}{t}(Y) \\
& =\left\langle W_{i}, \lim _{t \rightarrow 0} \frac{\exp (-t a d(X))-1}{t} Y\right\rangle \\
& =-\left\langle W_{i}, a d(X) Y\right\rangle=-\left\langle B\left(W_{i}, X\right), Y\right\rangle,
\end{aligned}
$$

where $L_{X}$ is the Lie derivative with respect to $X$. Namely, we have proved

$$
L_{X} \omega_{W_{i}}=-\omega_{B\left(W_{i}, X\right)}
$$

Let $\left\{C_{j k}^{i} ; i, j, k=1, \cdots, n\right\}$ be the structure-constants of $G$ with respect to the basis $\left\{W_{1}, \cdots, W_{n}\right\}$. Then

$$
B\left(W_{i}, X\right)=\sum_{j, k=1}^{n} \alpha_{j} C_{j k}^{i} W_{k},
$$

where

$$
X=\sum_{j=1}^{n} \alpha_{j} W_{j}
$$


By the expression of $\Omega$ we see that

$$
\begin{aligned}
-L_{X} \Omega & =\omega_{B\left(W_{1}, X\right)} \wedge \cdots \wedge \omega_{W_{n}}+\cdots+\omega_{W_{1}} \wedge \cdots \wedge \omega_{B\left(W_{n}, X\right)} \\
& =\left(\sum_{i, j=1}^{n} \alpha_{j} C_{j i}^{i}\right) \Omega=(\operatorname{Tr} \operatorname{ad}(X)) \Omega .
\end{aligned}
$$

Hence by the assumption 4) we obtain

$$
\operatorname{div} X=0 \text {. }
$$

Thus the proof is completed.

(G.E.D.)

THEOREM 3. The left-invariant vector field $X$ on PSL (2.R) which induces a $C$-flow for left-invariant Riemannian metric on PSL (2.R) is expressed in the form

$$
X=\xi_{1} X_{1}+\xi_{2} X_{2}+\xi_{3} X_{3}, \xi_{2}^{2}-\xi_{3}^{2}-\xi_{1} \xi_{3}>0,
$$

where $X_{1}, X_{2}, X_{3}$ are left-invariant vector fields defined in $\S 2$.

(Proof) Let $X$ be an element of the Lie algebra of PSL (2.R);

$$
X=\xi_{1} X_{1}+\xi_{2} X_{2}+\xi_{3} X_{3}
$$

Then

$$
a d(X)=\left(\begin{array}{ccc}
2 \xi_{2} & -2 \xi_{1}-4 \xi_{3} & 4 \xi_{2} \\
\xi_{3} & 0 & -\xi_{1} \\
0 & 2 \xi_{3} & -2 \xi_{2}
\end{array}\right)
$$

Hence the characteristic equation is expressed in the form

$$
\operatorname{det}(a d(X)-\lambda E)=-\lambda^{3}-4\left(\xi_{3} \xi_{1}-\xi_{2}^{2}+\xi_{3}^{2}\right) \lambda=0,
$$

where $E$ is the unit matrix. Hence, for $X$ to satisty the conditions 1),2), 3) of Theorem 2,

$$
\xi_{2}^{2}-\xi_{3}^{2}-\xi_{1} \xi_{3}>0
$$

is necessary and sufficient.

It is noted that, since $\operatorname{PSL}(2 . R)$ is a simple Lie group, the condition 4) holds for any left-invariant vector field on $P S L(2 . R)$. Thus the theorem is proved.

Corollary 3.1. Each instable stationary point described in Theorem 1 induces a C-flow on PSL (2.R) for any left-invariant Riemannian metric. 
TheOREM 4. The semi-semple real Lie algebra \&s which contains an element satisfying the conditions 1$), 2), 3), 4)$ of Theorem 2 is isomorphic to $\mathfrak{I} \mathfrak{l}(2 . R)$.

(Proof) The condition 1) implies that

$$
\operatorname{rank} \mathbb{S}=1 .
$$

Hence the complexification $\mathscr{S S}^{c}$ of $\mathscr{S}$ is a simple Lie algebra with rank 1. Appealing to Cartan's classification of simple Lie algebras over complex numbers $C$, we see that $\mathbb{S S}^{c}$ is isomorphic to $\mathfrak{I I}(2 . C)$. Hence $\mathbb{S}$ is a 3dimensional simple Lie algebra, and therefore $\mathbb{E S}$ is isomorphic to $\mathfrak{I I}(2 . R)$ or $\mathfrak{g} \mathfrak{D}(3 . R)$. On the other hand, the fact that the group is non-compact shows that $\&$ sf can not be isomorphic to $\mathfrak{S}_{\mathfrak{D}}(3 . R)$. Therefore, from Theorem 3 , \&s must be isomorphic to $\mathfrak{g l}(2 . R)$.

\section{§4. Further discussions on the $C$-flows on the unitary tangent} bundle $T_{1} L$ of the Lobachevsky-plane $L$.

In this section we discuss what movement is given on $T_{1} L$ by the $C$ flow described in Theorem 3.

Let us denote by $\left\{\varphi_{t} ; t \in R\right\}$ the geodesic flow on the Lobachevsky-plane L. Then we have

Proposition 4.1. Let $\Phi$ be the diffeomorphism of $T_{1} L$ onto PSL (2.R) described in \$2. Then we have

$$
\Phi \cdot \varphi_{t} \cdot \Phi^{-1}=\operatorname{Exp} \frac{t}{2} X_{2}
$$

where $X_{2}$ is the element of the Lie algebra $\mathfrak{g l}(2 . R)$ given in $\S 2$.

(Proof) Recall the definition of $v_{0}$, and define $\left\{g_{t} \in P S L(2 . R) ; t \in R\right.$. $\}$ as follows:

$$
\Phi\left(\varphi_{t} v_{0}\right)=g_{t}
$$

Then, we have for $t, s \in R$,

$$
\begin{aligned}
g_{t+s} & =\Phi\left(\varphi_{t+s} v_{0}\right)=\Phi\left(\varphi_{t} \varphi_{s} v_{0}\right)=\Phi\left(\varphi_{t} g_{s_{*}} v_{0}\right) \\
& =\Phi\left(g_{s_{*}} \varphi_{t} v_{0}\right)=\Phi\left(g_{s_{*}} g_{t *} v_{0}\right)=g_{s} \cdot g_{t} .
\end{aligned}
$$

Hence there exists $X \in \mathfrak{F l}(2 . R)$ such that

$$
g_{t}=\exp t X
$$


For any element $g \in P S L(2 . R)$, the relations

$$
\Phi \cdot \varphi_{t} \cdot \Phi^{-1}(g)=\Phi\left(\varphi_{t} g_{*} v_{0}\right)=\Phi\left(g_{*} g_{t_{*}} v_{0}\right)=g \cdot g_{t}=R_{g_{t}}(g)
$$

prove that

$$
\Phi \cdot \varphi_{t} \cdot \Phi^{-1}=\operatorname{Exp} t X
$$

On the other hand, the following formulas are easily obtained:

$$
x\left(\varphi_{t} v_{0}\right)=0, y\left(\varphi_{t} v_{0}\right)=e^{t}, \theta\left(\varphi_{t} v_{0}\right)=0,
$$

where $(x, y, \theta)$ is the local coordinate in $T_{1} L$. Hence by Proposition 2.1,

$$
\Phi\left(\varphi_{t} v_{0}\right)=g_{t}=\left(0, e^{t}, 0\right),
$$

or equivalently,

$$
X_{e}=\left(\frac{\partial}{\partial y}\right)_{e}=\frac{1}{2}\left(X_{2}\right)_{e} .
$$

Consequently we obtain

$$
\Phi \cdot \varphi_{t} \cdot \Phi^{-1}=\operatorname{Exp} \frac{t}{2} X_{2} .
$$

Let us denote by $T_{\alpha}(0 \leq \alpha<2 \pi)$ the diffeomorphism of $T_{1} L$ onto itself given by

$$
T_{\alpha} ; T_{1} L \ni(x, y, \theta) \longrightarrow(x, y, \theta+\alpha) \in T_{1} L,
$$

and denote by $H$ the upper half-plane. Then we obtain

Theorem 5.

i) Assume that

$$
2 \xi_{3}+\xi_{1} \neq 0, \xi_{2}^{2}-\xi_{3}^{2}-\xi_{1} \xi_{3}>0 .
$$

Then $\operatorname{Exp} t \Phi_{*}^{-1}\left(\xi_{1} X_{1}+\xi_{2} X_{2}+\xi_{3} X_{3}\right)$ is a $C$-flow on $T_{1} L$, but it cannot be a geodesic flow with respect to any Riemannian metric on $\mathrm{H}$.

ii) Assume that

$$
2 \xi_{3}+\xi_{1}=0, \xi_{2}^{2}+\frac{1}{4} \xi_{1}^{2}>0 .
$$

Then we have

$$
\operatorname{Exp} t \Phi_{*}^{-1}\left(\xi_{1} X_{1}+\xi_{2} X_{2}+\xi_{3} X_{3}\right)=T_{\alpha} \cdot \varphi_{\rho t} \cdot T_{-\alpha},
$$


where

$$
\xi_{1}=\rho \sin \alpha, 2 \xi_{2}=\rho \cos \alpha, \rho>0,0 \leqq \alpha<2 \pi .
$$

(Proof) Let us now express $X_{1}, X_{2}, X_{3}$ in terms of the local coordinates $(x, y, \theta)$ in $T_{1} L$;

$$
(*)\left\{\begin{array}{l}
X_{1}=y \cos 2 \theta \frac{\partial}{\partial x}+2 y \sin 2 \theta \frac{\partial}{\partial y}+\sin ^{2} \theta \frac{\partial}{\partial \theta} \\
X_{2}=-2 y \sin 2 \theta \frac{\partial}{\partial x}+2 y \cos 2 \theta \frac{\partial}{\partial y}+\sin 2 \theta \frac{\partial}{\partial \theta} \\
X_{3}=\frac{\partial}{\partial \theta}
\end{array}\right.
$$

By Proposition 2.1, $\Phi_{*}^{-1}\left(\xi_{1} X_{1}+\xi_{2} X_{2}+\xi_{3} X_{3}\right)$ induces the following differential equations on $T_{1} L$;

$$
\left\{\begin{array}{l}
\dot{x}=\xi_{1} y \cos \theta-2 \xi_{2} y \sin \theta \\
\dot{y}=\xi_{1} y \sin \theta+2 \xi_{2} y \cos \theta \\
\dot{\theta}=2\left(\xi_{1} \sin ^{2} \frac{\theta}{2}+\xi_{2} \sin \theta+\xi_{3}\right),
\end{array}\right.
$$

which imply

$$
\left\{\begin{array}{l}
\ddot{x}-\frac{2 \dot{x} \dot{y}}{y}=-\left(2 \xi_{3}+\xi_{1}\right) \dot{y} \\
\ddot{y}+\frac{\dot{x}^{2}-\dot{y}^{2}}{y}=\left(2 \xi_{3}+\xi_{1}\right) \dot{x} .
\end{array}\right.
$$

These prove the case i).

We now assume

$$
2 \xi_{3}+\xi_{1}=0
$$

Then follows

$$
\xi_{2}^{2}-\xi_{3}^{2}-\xi_{1} \xi_{3}=\xi_{2}^{2}+\frac{1}{4} \xi_{1}^{2}>0 .
$$

Let us introduce new parameters $\rho, \alpha$ as follows:

$$
2 \xi_{2}=\rho \cos \alpha, \quad \xi_{1}=\rho \sin \alpha, \rho>0,0 \leq \alpha<2 \pi .
$$

Then, by the formulas (*) we have 


$$
\begin{gathered}
\Phi_{*}^{-1}\left(\xi_{1} X_{1}+\xi_{2} X_{2}+\xi_{3} X_{3}\right)=-\rho y \sin (\theta-\alpha) \frac{\partial}{\partial x}+o y \cos (\theta-\alpha) \frac{\partial}{\partial y} \\
+\rho \sin (\theta-\alpha) \frac{\partial}{\partial \theta} .
\end{gathered}
$$

On the other hand, by Proposition 4.1. the infinitesimal transformation of $\varphi_{t}$ is expressed in the form

$$
\Phi_{*}^{-1} X=\frac{1}{2} \Phi_{*}^{-1} X_{2}=-y \sin \theta \frac{\partial}{\partial x}+y \cos \theta \frac{\partial}{\partial y}+\sin \theta \frac{\partial}{\partial \theta} .
$$

Therefore we have

$$
\Phi_{*}^{-1}\left(\xi_{1} X_{1}+\xi_{2} X_{2}+\xi_{3} X_{3}\right)=T_{\alpha^{*}} \rho \Phi_{*}^{-1} X
$$

We exponentiate both sides to obtain

$$
\begin{aligned}
\operatorname{Exp} t \Phi_{*}^{-1}\left(\xi_{1} X_{1}+\xi_{2} X_{2}+\xi_{3} X_{3}\right) & =\operatorname{Exp} t T_{\alpha^{*}} o \Phi_{*}^{-1} X \\
& =T_{\alpha} \cdot \operatorname{Exp} \rho t \Phi_{*}^{-1} X \cdot T_{\alpha}^{-1} \\
& =T_{\alpha} \cdot \varphi_{\rho t} \cdot T_{-\alpha},
\end{aligned}
$$

which proves the case ii).

(Q.E.D.)

\section{REFERENCES}

[1] V.I. Arnol'd: Sur la géométrie différentielle des groupes de Lie de dimension infinié et ses applications à l'hydrodynamique des fluides parfaits. Ann. Inst. Fourier (1966) XVI. (1) p. 319-363.

[2] V.I. Aronl'd-A. Avez: Problèmes ergodiques de la mécanique classique. Gauthier-Villars. Paris (1967).

[3] E.T. Whittaker: A treatise on the analytical dynamics of particles and rigid bodies. Cambridge. (1952).

Mathematical Institute, Nagoya University 